\title{
BMJ Open Study protocol of the European Urban Burden of Disease Project: a health impact assessment study
}

Mark J Nieuwenhuijsen (D) , ${ }^{1}$ Jose Barrera-Gómez, ${ }^{1}$ Xavier Basagaña, ${ }^{1,2}$ Marta Cirach, ${ }^{1}$ Carolyn Daher, ${ }^{1}$ Maria Foraster Pulido, ${ }^{1,3}$ Tamara lungman, ${ }^{1}$ Antonio Gasparrini, ${ }^{4}$ Gerard Hoek, ${ }^{5}$ Kees de Hoogh, ${ }^{6,7}$ Sasha Khomenko, ${ }^{1}$ Haneen Khreis, ${ }^{8}$ Audrey de Nazelle, ${ }^{9}$ Ana Ramos, ${ }^{1}$ David Rojas-Rueda (1) ,10 Evelise Pereira Barboza (D) , ${ }^{1}$ Marko Tainio, ${ }^{11,12}$ Meelan Thondoo, ${ }^{1}$ Cathryn Tonne, ${ }^{1}$ James Woodcock, ${ }^{13} \mathrm{~N}^{\text {Mueller }}{ }^{1}$

To cite: Nieuwenhuijsen MJ, Barrera-Gómez J, Basagaña X, et al. Study protocol of the European Urban Burden of Disease Project: a health impact assessment study. BMJ Open 2022;12:e054270. doi:10.1136/ bmjopen-2021-054270

- Prepublication history for this paper is available online. To view these files, please visit the journal online (http://dx.doi. org/10.1136/bmjopen-2021054270).

Received 09 June 2021 Accepted 24 December 2021

Check for updates

(C) Author(s) (or their employer(s)) 2022. Re-use permitted under CC BY-NC. No commercial re-use. See rights and permissions. Published by BMJ.

For numbered affiliations see end of article.

\section{Correspondence to}

Professor Mark J

Nieuwenhuijsen;

mark.nieuwenhuijsen@isglobal. org

\section{ABSTRACT}

Introduction Cities have long been known to be society's predominant engine of innovation and wealth creation, yet they are also hotspots of pollution and disease partly due to current urban and transport practices. The aim of the European Urban Burden of Disease project is to evaluate the health burden and its determinants related to current and future potential urban and transport planning practices and related exposures in European cities and make this evidence available for policy and decision making for healthy and sustainable futures.

Methods and analysis Drawing on an established comparative risk assessment methodology (ie, Urban and Transport Planning Health Impact Assessment) tool), in nearly 1000 European cities we will (1) quantify the health impacts of current urban and transport planning related exposures (eg, air pollution, noise, excess heat, lack of green space) (2) and evaluate the relationship between current levels of exposure, health impacts and city characteristics (eg, size, density, design, mobility) (3) rank and compare the cities based on exposure levels and the health impacts, (4) in a number of selected cities assess in-depth the linkages between urban and transport planning, environment, physical activity and health, and model the health impacts of alternative and realistic urban and transport planning scenarios, and, finally, (5) construct a healthy city index and set up an effective knowledge translation hub to generate impact in society and policy. Ethics and dissemination All data to be used in the project are publicly available data and do not need ethics approval. We will request consent for personal data on opinions and views and create data agreements for those providing information on current and future urban and transport planning scenarios.

For dissemination and to generate impact, we will create a knowledge translation hub with information tailored to various stakeholders.

\section{INTRODUCTION}

Over $50 \%$ of people worldwide live in urban areas and this number is expected to increase up to $70 \%-80 \%$ over the next 20 years. ${ }^{1}$ The

\section{Strengths and limitations of this study}

- For the first time, the health impacts of current urban and transport planning practices and relationship with city characteristics in nearly 1000 European cities will be quantified.

- The linkages between urban and transport planning, environment, physical activity and health will be assessed in depth, and the health impacts of alternative urban and transport planning scenarios in cities will be modelled.

- We will employ a robust comparative risk assessment methodology (Urban and Transport Planning Health Impact Assessment) using state of the art input data on, for example, exposure response relationships to quantify related health impacts.

- Our analyses will be performed at high resolution (ie, grid-cell level with $250 \mathrm{~m} \times 250 \mathrm{~m}$ resolution), and the results will be estimated and compared within and between the cities, contributing to the design of evidence-based urban policies.

- The work is limited by the quantity, quality and comparability of some of the available city level exposure and health data, and the need to make assumptions where causal inference or specific guidelines is lacking.

United Nations has also recently projected that nearly all global population growth from 2016 to 2030 will be absorbed by cities, adding about 1.1 billion new urbanites over the next 14 years. In Europe, more than $70 \%$ of people live in urban areas. Cities have long been known to be society's predominant engine of innovation and wealth creation, yet they are also a main source of pollution and disease. ${ }^{2}$ Cities in Europe provide good opportunities for policy change as city councils have direct local accountability and have more agile governance structures compared 
than national governments. They also set local urban and transport policies.

Appropriate and efficient urban planning and transport systems are essential for cities to thrive, but evidence suggests that current urban and transport planning practices can cause increased exposure to air pollution and noise, heat islands and a lack of green space and physical inactivity that have detrimental effects on health such as increasing morbidity and premature mortality. ${ }^{3}{ }^{4}$ Air pollution, ${ }^{56}$ noise $^{78}$ and excess heat ${ }^{9}$ cause increased morbidity and premature mortality, green space has both positive and negative health effects ${ }^{10} 11$ and physical activity ${ }^{12}$ is associated with longevity and physical and mental health. Currently, there is considerable variability within and between cities in levels of environmental exposures, such as air pollution, noise, excess heat and green space. There are cities with low levels of air pollution and noise, and sufficient green space for favourable health outcomes evidencing that a reduction in harmful and an increase in beneficial exposures through targeted policy action is possible. ${ }^{34}$

Quantitative health impact assessment (HIA) or similar methodologies, based on the comparative risk assessment approach, are becoming increasingly used, but until now, estimates have mainly been produced on national or regional levels (eg, Global Burden of Disease (GBD)). ${ }^{13}$ Physical inactivity and ambient air pollution are estimated to cause more than three million and four million premature deaths respectively each year worldwide, ranking them among the leading risk factors in the GBD study. Furthermore, a number of country wide HIAs have been conducted on a number of environmental risk factors, for example, for benzene, dioxins, secondhand smoke, formaldehyde, lead, traffic noise, ozone, particulate matter (PM2.5) and radon, ${ }^{14}$ traffic emissions,${ }^{15}$ traffic incidents, air pollution and noise, ${ }^{16}$ annoyance and sleep disturbance by noise ${ }^{17}$ and active transportation. ${ }^{18-20}$

\section{Knowledge gaps}

National and regional estimates, however, are often less useful for city councils who have to take decisions on local scales with direct impacts on city dwellers daily lives. They want to have local estimates of health impacts, backed by scientific evidence, to base their decisions and solutions on. HIAs have suggested to be useful in this regard although often hard to prove because of the multifactoral process leading to policy decisions. Therefore, there is the need to provide these health impacts on a city scale. There are some urban and transport related HIAs on city level for example for noise in Madrid, ${ }^{21}$ air pollution in Basel $^{22}$ and Malmo, ${ }^{23}$ alternative land scenarios in London and Delhi ${ }^{24}$ alternative land-use and transport policy initiatives in six cities, ${ }^{25}$ various exposures in Barcelona ${ }^{2627}$ and Port Louis (Mauritius), ${ }^{28}$ noise $^{29}$ and air pollution ${ }^{30}$ in Houston and the Superblock model in Barcelona ${ }^{31}$ Furthermore, some studies showed variation in health impacts of environmental exposures by ethnic or socioeconomic status (SES) in Bradford, Vienna,
Madrid and Barcelona. ${ }^{32} 33$ While such efforts are available sporadically, there is a lack of a more systematic assessment of these exposures, and their distribution among the population, for many European cities at the same time. While we have estimates for a few cities, we lack harmonised data at the city level, as the ones done at the country level.

\section{Aims}

The aim of the European Urban Burden of Disease project is to evaluate the health burden and its determinants related to current and future potential urban and transport planning practices and related exposures in European cities. As part of the research, in nearly 1000 European cities we will (1) quantify the health impacts of current urban and transport planning related exposures (eg, air pollution, noise, excess heat, green space), (2) evaluate the relationship between current levels of exposure and city characteristics (eg, size, density, design, mobility), (3) rank and compare the cities based on exposure levels and health impacts, and (4) in a number of selected cities assess in-depth the linkages between urban and transport planning, environment, physical activity and health, and model the health impacts of alternative and realistic urban and transport planning scenarios, and, finally (5) construct a healthy city index and set up an effective knowledge translation hub to generate impact in society and policy.

\section{METHODS AND ANALYSES}

The project is organised into different components. First, we will gather information and develop the overall framework, model and tool to be used in the project, based on our previous experience and help of external advisers. Second, we will collect all the city level input data (eg, environmental exposure, city characteristics, demographic and health data) for the HIA modelling. Third, we will conduct the HIAs and produce health impact estimates, provide a comparison of cities and evaluate the association with city characteristics. This will be followed by in-depth HIA of alternative urban and transport planning scenarios in a number of selected cities. Finally, we will also construct a healthy city index and set up an effective knowledge translation hub.

\section{HIA framework and model development}

We will develop a HIA framework, model and tool to estimate mortality and morbidity impacts of urban and transport-related exposures and lifestyles including air pollution, noise, excess heat, green space, traffic injuries and physical activity for European cities. We will focus on these exposures as they have been identified as among the strongest health determinants, best quantifiable and easiest obtainable exposures related to the core work scopes of urban and transport planning in Europe, but others may be included depending on the outcome of an initial expert consultation. 


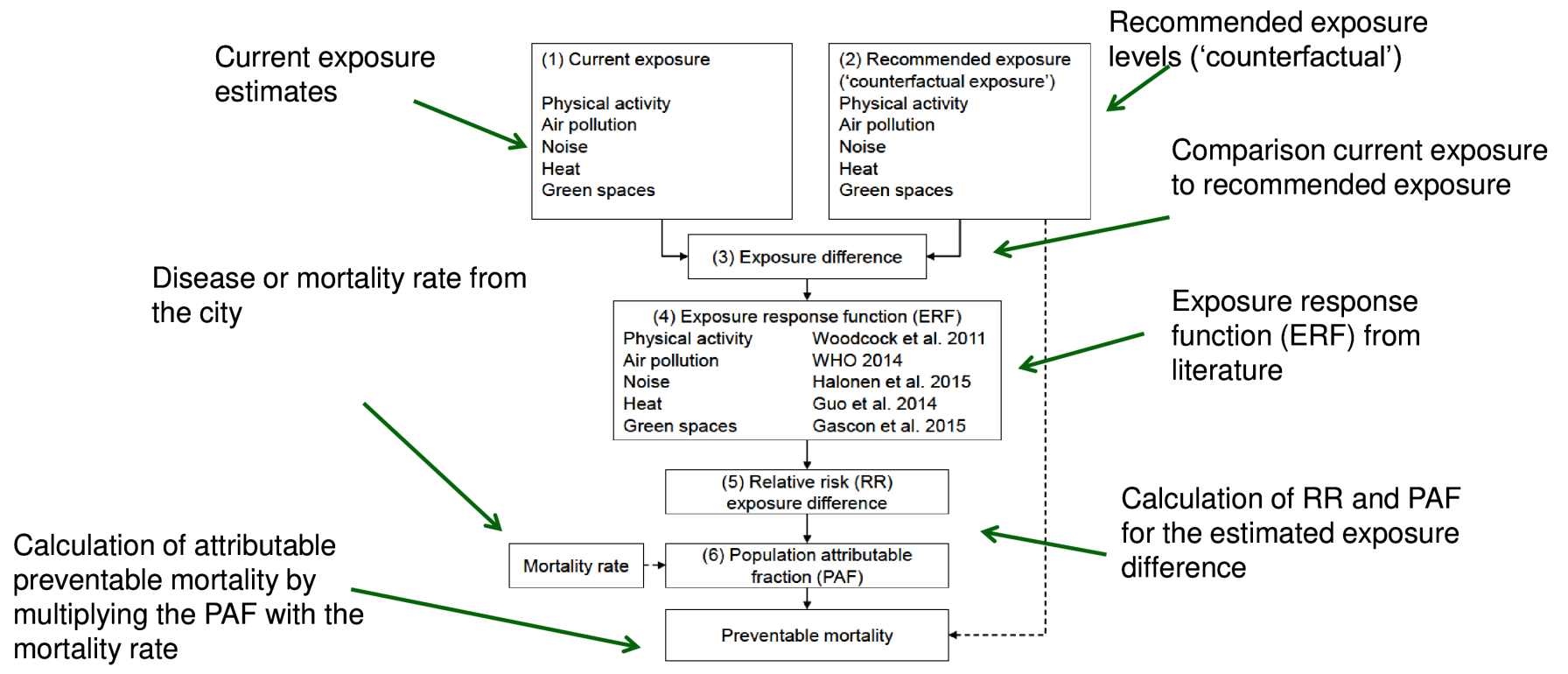

Mueller et al EHP 2017; 125: 89-96

Figure 1 Urban and Transport Planning Health Impact Assessment (UTOPHIA) tool.

We will develop a conceptual framework and frame the issues based on our previous work (Urban and Transport Planning Health Impact Assessment, UTOPHIA model). ${ }^{26} 2734-36$ The UTOPHIA tool, which is based on our previous work, will be further developed with algorithms that will be implemented in the statistical software $\mathrm{R}$ (figure 1). We will include innovative uncertainty analysis to overcome drawbacks of HIA that draw on assumptions where causal inference is uncertain. Improved uncertainty analysis will provide more robust uncertainty intervals in the health impact estimates. This will help decide where the research efforts should be focus in order to reduce the uncertainty and continuously improve the applied methodology.

We will select exposure-response relationships for mortality and morbidity for the exposures of interest from the literature, preferably from meta-analyses. We will conduct a systematic review for exposure-response functions for the different exposures and health outcomes, and conduct new meta-analyses if needed. When available, we will work with exposure-response functions recommended by authoritative bodies such as the WHO, for example for air pollution, ${ }^{63738}$ and noise, ${ }^{39}$ or those used in previous peer-reviewed HIAs. ${ }^{26}{ }^{27}$ We will use criteria to obtain exposure-response functions following the GBD approach, ${ }^{13}$ which take into account the importance of each risk factor to either disease burden, policy or both; the availability of sufficient data to estimate risk factor exposure; evidence from epidemiological studies supporting a causal relation between the risk factor and the outcome and available data to estimate effect sizes per unit of exposure increase; and evidence that these effects can be applied to a general population.
We will develop and implement the models and data into an integrated web-based software package as a tool that can be applied to and by cities. We will implement the above information in a relatively easy-to-use software package, and produce a comprehensive user guide to facilitate the usage of the tool by city authorities and other researchers and stakeholders. We are aiming for a web based version that is easily accessible for those who want to use it like the WHO HEAT and AirQ +tools but with a higher level of precision, for example, taking into account geographical variation and uncertainties. We will provide training workshops and a detailed user manual.

\section{Data collection}

We will obtain and create a database for city spatial boundary data, urban and transport planning related exposures, including air pollution, noise, temperature, green space and city characteristics (eg, demographics, size, density, land use, connectivity, design, mobility, SES related indicators) for nearly 1000 cities in Europe (figure 2). We will use the European cities definition and spatial boundary data from the Urban Audit 2018 database. ${ }^{40}$ The dataset contains 980 cities and 49 greater cities in 31 European countries. The 49 greater cities cover 161 cities either by representing a city of bigger area than the city or by constituting a combination of several cities. We will exclude Saint Denis (Réunion) and Fort-deFrance (Martinique) due to their location outside of the European study area, and perform the analyses for a final dataset of 978 cities and 49 greater cities. Furthermore, we will collect and estimate information at $250 \mathrm{~m}$ x $250 \mathrm{~m}$ resolution; on population numbers from the Global Human Settlement Layer, mortality at the city level from 

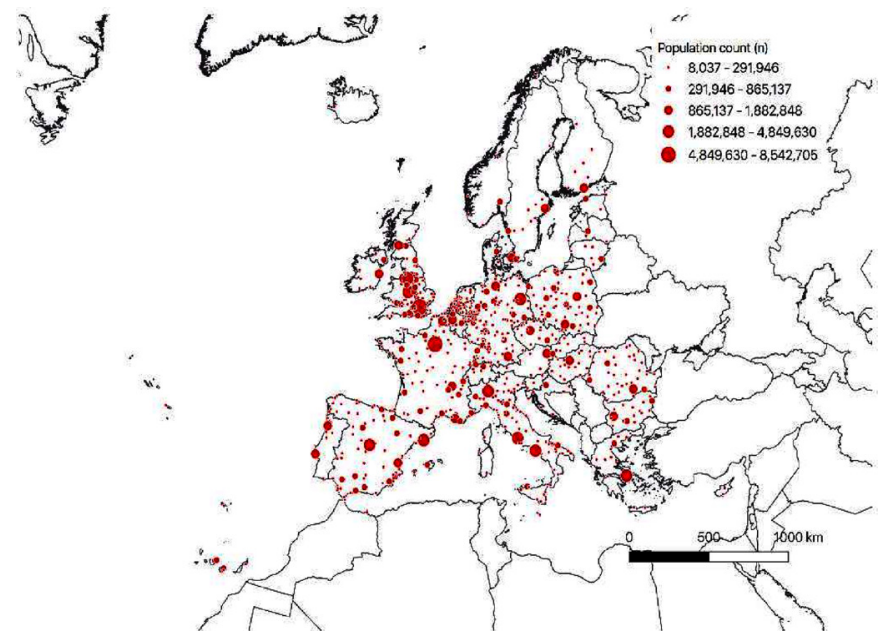

Figure 2 Cities in Europe in the study.

Eurostat, morbidity at the city level from local databases, and relevant city characteristics data from the cities with publicly available data. For the nearly 1000 cities, we will focus on environmental exposures and mortality, as this is the only city specific information commonly available for so many cities. Moreover, the multiple urban and transport planning related exposures have consistently been associated with premature mortality, allowing for the comparison of magnitude of impacts. The target for data collection is from 2015 onwards.

We will develop a protocol and collect data for urban and transport planning related exposures including air pollution, noise, temperature and green space and spatial boundaries for the cities. Air pollution estimates will be obtained by using existing Land Use Regression model estimates for Europe (eg, ELAPSE) ${ }^{41}$ measurements from the European air pollution network (eg, Airbase) and new air pollution modelling work, using a combination of measurements and remote sensing data to produce high resolution air pollution estimates for cities. We will estimate the specific source contribution based on the distribution of the pollutants, local source apportionment studies conducted in the cities, data available in the literature ${ }^{4243}$, and/or other European research projects that are currently ongoing and with which we have agreed to share data (eg, GBD-MAPS, SHERPA cities). Cities with more than 100000 inhabitants are required to produce noise maps under European Noise Directive (END) (Directive 2002/49/EC). Noise estimates will be obtained from either the European Environment Agency repository for noise under the END and/or from local country/regional sources, depending on availability (available for around 500 cities only). Green space exposure will be obtained using land use maps (Urban Atlas, UK and Corine Land Cover Maps) and remote sensing (NDVI, Landsat). Temperature data will be obtained from the UrbClim model at a high resolution level and from daily gridded observational dataset (E-obs) at a low resolution level. Traffic accident and physical activity data will be collected from existing city-wide surveys and existing ad hoc research projects in the cities.

City characteristics (eg, size, urban form, density, land use, street design and connectivity) will be estimated and extracted from geographical information systems (GIS) databases like Open Street Map (OSM) and other sources such as Urban Atlas and Copernicus. The environmental data will be collected at $250 \mathrm{~m}$ grid cell level or equivalent level, to be consistent with the population grid dataset which is the more detailed data available for Europe so far and that allows to obtain the spatial distribution in the cities. All the information will be stored in GIS databases. Specific protocols and procedures will be put in place to guarantee the data harmonisation and, therefore, its comparability, following similar protocols and procedures from previous projects.

We will develop a protocol and collect data on population counts, mortality (all cities) and morbidity (selected cities) by age and sex. Mortality and morbidity data will be obtained from the city councils and other available sources (morbidity) and Eurostat (mortality). ${ }^{44}$ Total all-cause mortality counts are available at the city level through Eurostat. Overall, when there are missing mortality counts, all-cause mortality counts will be estimated employing the corresponding NUTS3, NUTS2 or country-level all-cause mortality rates. The NUTS classification (Nomenclature of territorial units for statistics) is a hierarchical system for dividing up Europe based to different purposes. ${ }^{40}$ NUTS 2 correspond to basic regions within countries (ie, Autonomous Communities) and NUTS 3 refers to smaller regions (ie, Provinces) To calculate the number of natural-cause deaths by age group for each city and greater city we will retrieve NUTS3-level mortality counts by age group and NUTS2 and countrylevel mortality counts by age and cause of death. ${ }^{45}{ }^{46} \mathrm{We}$ will calculate the external deaths fractions (defined by the ICD-10 mortality codes V01-Y89) by age group and estimate the proportion of natural-cause deaths by age group at the NUTS3-level. These proportions will be then applied to the corresponding city-level total all-cause mortality counts.

We will develop an open database that can be accessed by the research community and stakeholders in the cities. The database will contain all the data collected and generated by the project and will be held at ISGlobal with easy access for other researchers, city planners and practitioners.

\section{Estimate the burden of disease}

We will estimate the mortality burden due to the selected exposures for the nearly 1000 cities and compare the cities by health impact magnitude. Furthermore, we will evaluate the health impacts in relation to city characteristics (eg, demographics, size, density, land use, connectivity, design, mobility, SES). We will conduct a quantitative HIA by combining information on all the above objectives and tasks and obtain estimates of the mortality burden related to the current urban and transport planning practices and 
their associated exposure levels in the cities. We will use a comparative risk assessment framework and the updated UTOPHIA tool. ${ }^{26}$ We will combine the information from the exposure estimates, exposure-response functions and background health data to produce burden of disease estimates in the different cities, expressed as the number of cases of premature mortality, percentage of the total mortality (TV), Years of life lost (YLLs), Years of living with disease (YLDs), Disability adjusted life years (DALYs).

Counterfactual exposures include, for example, meeting international health guidelines (eg, WHO exposure limits), local policy goals, and meeting exposure levels in the city with, for example, lowest level of air pollution or standards of cities that we consider to be doing well in terms of exposures. We will account for competing risks where necessary, using methods established by the GBD study. ${ }^{13}$ We will produce central tendency estimates, confidence intervals and ranges considering the uncertainties in the input data and HIA methodologies, that is, exposures, mortality rates, ERFs, etc.

We will make a ranking and comparison of cities by health impact of the environmental exposures. Furthermore, we will evaluate the health impacts in terms of city characteristics (eg, size, density, land use, street design and connectivity exposures) and explore the extent to which these characteristics are related to the health impact estimates. Initial analyses will focus on descriptive analyses and correlation, followed by regression and cluster analyses, and structural equation models, where appropriate. We will test the robustness and sensitivity of the models to input parameters by conducting sensitivity tests to address uncertainties. We will test the robustness of the model by varying the input parameters and testing the sensitivity of the output parameters to the input parameters. The work will build on the experience we have gained with conducting the pilot study in Barcelona ${ }^{26}{ }^{27}$ and published follow-up work for Bradford, Vienna, Barcelona and Madrid. ${ }^{32} 3347$ The work will be conducted at $250 \mathrm{~m} \times 250 \mathrm{~m}$ scale, taking into account the spatial distribution in the cities. We will produce online interfaces with the ranking of the cities by exposure levels and burden of disease to inform and raise awareness among policy-makers and relevant stakeholders and to guide them to formulate targeted policy actions (https:// isglobalranking.org/).

\section{In-depth assessment alternative urban and transport planning scenarios}

As part of the project, we will conduct an in-depth assessment of current status of urban and transport planning and develop alternative, sustainable and healthy scenarios for a number of cities (eg, Barcelona, Paris, Utrecht, Kiev). We will conduct an in-depth evaluation of current land use and transport practices in the cities and their respective planning for the future, using available routine data, surveys and meetings with stakeholders (around 10-20) inside and outside the local councils and assess in-depth the linkages between urban and transport planning, environment, physical activity and health. We will assess in detail the linkages between urban and transport planning, environment, physical activity and health, based on existing work and/or new analyses that need to be conducted. We will apply uniform analysis protocols to the data.

We will review the grey and peer-reviewed literature, reports from large consultancies (eg, KPMG, ARUP), international organisations (eg, UN-Habitat, WHO, OECD, EC, ICLEI, C40) and national governments on future city developments and trends in terms of land use, mobility patterns and technological changes, and people's perception, attitudes and behaviours towards those proposals. We will develop alternative, credible and realistic scenarios based on real life proposals in the cities and different assumptions for green, sustainable, equitable and healthy urban and transport planning, and compare and contrast the scenarios. Based on input from the workshops, politicians, decision makers, citizens and other stakeholders, we will develop three to five scenarios for each city, using the newly developed HIA methods, models and tools. Potential alternative urban and transport scenarios in the cities are e.g. change in compactness/density/intensity, changes in availability and quality of public and green space by changes in land use, other green interventions such as green roofs, changes in transport patterns such as mode shifts from cars towards public and active transportation, changes in vehicle fleet composition including fuels, vehicle emission standards and technology adaptation, for example, electric cars and autonomous vehicles. Selected scenarios may be different for each city as, for example, the land use, available transport systems, stakeholder needs and preferences and socioeconomic circumstances. We will aim to fully integrate the information obtained from relevant stakeholders, into the scenario development to create feasible and acceptable scenarios and provide the data for input into the models. We will do some additional exposure modelling, for example, using Atmospheric Dispersion Modelling System for air pollution, ${ }^{48}$ TRANEX, ${ }^{49}$ Computer Aided Noise Abatement (Cadna) (noise) and other software, where needed, and also include traffic accidents, for which information will be obtained from the cities.

We will develop an urban health equity assessment of the scenarios in the cities. The health equity assessment will identify vulnerable population groups, with the aim to narrow the health gap, and reduce inequities throughout the whole population. We will focus on SES as one of the main indicators for the assessment, but may include others where appropriate for the cities involved. We will conduct HIA, as described above, by combining information on all the above objectives and tasks and obtaining estimates of the burden of disease related to the current situation and for the future scenarios for the cities.

Finally, we will construct a healthy city index, bringing together the various aspect of what we learnt about health impacts of the exposures, city characteristics and in-depth 
modelling. The work will start with a review of the literature $^{50}$ and enhanced by the relative importance of health impacts as of the various exposures and their relation to city characteristics.

\section{Challenges}

The main challenges of the work are developing and applying the appropriate HIA framework and model(s), obtaining the (harmonised) input and output data for the HIA for so many cities, addressing uncertainty (eg, in population, exposure, health and exposure-response functions), developing and modelling the alternative healthy urban and transport planning scenarios with good input data, and developing and implementing an effective knowledge translation approach. Finally, as part of the work many assumptions need to be made and these need to be taken based on best available evidence and be well documented and expressed in the work.

\section{Utility of the project and data}

The project has many benefits including that it centralises urban health data in one location, makes available results for single or multiple cities in Europe, supports action and evidence-base policy making, develops new methods, identifies data gaps and needs, helps prioritisation to decision-makers, supports joint efforts for data collection and harmonisation, there is continuing exchange, feedback and improvement, provides high-resolution data and knowledge translation and supports stakeholder communication

\section{Patient and Public Involvement}

No patient involvement

\section{Progress to date}

An initial proof-of-concept assessment has been conducted. We conducted a quantitative HIA for the year 2015 to estimate the effect of air pollution exposure (PM2.5 and NO2) on natural-cause mortality for adult residents (aged $\geq 20$ years) in 969 cities and 47 greater cities in Europe. ${ }^{47}$ The study found that compliance with WHO air pollution guidelines could prevent 51213 (95\% CI 34036 to 68 682) deaths per year for PM2.5 exposure and $900(0-2476)$ deaths per year for NO2 exposure. The reduction of air pollution to the lowest measured concentrations could prevent 124729 (83 332-166 535) deaths per year for PM2.5 exposure and 79435 (0-215 165) deaths per year for NO2 exposure.

\section{Ethics and dissemination}

No ethics approval is needed for the work because the work uses mainly public available data. No new personal health data will be collected. Where we collect ask people provide opinions, we will ask them to sign a data processing form that also addressed data privacy issues.

For dissemination and to generate impact we will create a knowledge translation hub with information tailored to various stakeholders. This will allow dynamic, multilevel exchange of information and feedback throughout the project lifecycle. The hub will foster communities of practice across disciplines and stakeholders, and will help ensure the relevance of outputs, as well uptake and dissemination of results beyond the project duration. We will map and engage the existing relevant platforms, networks and stakeholders in the local city councils (urban and transport planners, environmental and health professionals, education sector), business, NGOs, research centres and citizen groups and stakeholders from national and international organisations (eg, WHO, UN-Habitat, ICLEI, UCLG, POLIS network) related to urban and transport planning, environment, public health and economy to create a stakeholder hub. Once mapped, partners will be engaged through kick-off events in each of the cities and targeted communication and outreach activities. Through the hub, stakeholders will provide key inputs to inform the HIA exercise.

We will develop a communication and translation strategy including a website and social media (eg, Facebook, Twitter) to disseminate information about the project, results and engage with relevant stakeholders and other interested parties. Capitalising on our capacity for knowledge translation, in addition to scientific papers for specialist high-impact journals, we will prepare content for popular science articles, news media articles and blogs, and conduct radio and TV interviews. We will create visual materials such as infographics, webinars, photo essays, podcasts and short videos that can communicate with diverse audiences about the project process and its results. We work on capacity building and short training in HIA for the interested stakeholders especially for the tools that are developed. We will prepare policy briefs for decision makers at the technical and political levels. For all translation activities, we will link with existing networks from project stakeholders to disseminate information. Finally, we will produce rankings of cities in terms of health impacts of urban and transport planning scenarios and similar outputs to attract attention. The ranking will also enable city stakeholders to compare health-related achievements over time and improve urban interventions and policies.

We will produce online interfaces with the ranking of the cities by exposure levels and burden of disease to inform and raise awareness among policy-makers and relevant stakeholders and to guide them to formulate targeted policy actions (https://isglobalranking.org/).

\footnotetext{
Author affiliations

${ }^{1}$ Barcelona Institute for Global Health, Barcelona, Spain

${ }^{2}$ Pompeu Fabra University Faculty of Health and Life Sciences, Barcelona, Spain

${ }^{3}$ Ramon Llull University, Barcelona, Spain

${ }^{4}$ London School of Hygiene \& Tropical Medicine, London, UK

${ }^{5}$ IRAS, Utrecht University Faculty of Veterinary Medicine, Utrecht, Netherlands

${ }^{6}$ Swiss Tropical and Public Health Institute, Basel, Switzerland

${ }^{7}$ Basel University Faculty of Philosophy and Natural Sciences, Basel, Switzerland

${ }^{8}$ Texas A\&M University System, College Station, Texas, USA

${ }^{9}$ Imperial College London, London, UK

${ }^{10}$ Colorado State University System, Fort Collins, Colorado, USA

${ }^{11}$ SYKE, Helsinki, Finland

${ }^{12}$ Polish Academy of Sciences, Warszawa, Poland
} 
${ }^{13} \mathrm{PH}$, Cambridge University, Cambridge, UK

Twitter Jose Barrera-Gómez @overdispersion and David Rojas-Rueda @drrbcn Contributors MJN conceptualised the project and drafted the manuscript. MJN, $\mathrm{JB}, \mathrm{XB}, \mathrm{MC}, \mathrm{CD}, \mathrm{MFP}, \mathrm{TI}, \mathrm{AG}, \mathrm{GH}, \mathrm{KdH}$, SK, HK, AdN, AR, DR-R, EPB, MTa, MTh, $\mathrm{CT}$, JW and NM contributed to collection, and drafting, editing, and reviewing the manuscript. MJN, JB, XB, MC, CD, MFP, TI, AG, GH, KdH, SK, HK, AdN, AR, DR-R, EPB, MTa, MTh, CT, JW and NM read the final version of this manuscript, approved it for submission for publication, and agreed to be accountable for all aspects of the work.

Funding This project receives funding and support from SURREAL through the European Union's Horizon 2020 research and innovation programme under grant agreement No 956780, GoGreenRoutes through the European Union's Horizon 2020 research and innovation programme under grant agreement № 869764, EXPANSE through the European Union's Horizon 2020 research and innovation programme under grant agreement № 874627 and a Fellowship from the Spanish Ministry of Science and Innovation through the ayudas para la formación de profesorado universitario 2020-2024 doctoral funding.

Map disclaimer The inclusion of any map (including the depiction of any boundaries therein), or of any geographic or locational reference, does not imply the expression of any opinion whatsoever on the part of BMJ concerning the legal status of any country, territory, jurisdiction or area or of its authorities. Any such expression remains solely that of the relevant source and is not endorsed by BMJ. Maps are provided without any warranty of any kind, either express or implied.

Competing interests None declared.

Patient and public involvement Patients and/or the public were not involved in the design, or conduct, or reporting, or dissemination plans of this research.

Patient consent for publication Not applicable.

Provenance and peer review Not commissioned; externally peer reviewed.

Open access This is an open access article distributed in accordance with the Creative Commons Attribution Non Commercial (CC BY-NC 4.0) license, which permits others to distribute, remix, adapt, build upon this work non-commercially, and license their derivative works on different terms, provided the original work is properly cited, appropriate credit is given, any changes made indicated, and the use is non-commercial. See: http://creativecommons.org/licenses/by-nc/4.0/.

\section{ORCID iDs}

Mark J Nieuwenhuijsen http://orcid.org/0000-0001-9461-7981

David Rojas-Rueda http://orcid.org/0000-0001-5854-2484

Evelise Pereira Barboza http://orcid.org/0000-0002-2995-7418

\section{REFERENCES}

1 United Nations. World urbanization prospects. The 2014 revision, highlights, 2014. Available: http://esa.un.org/unpd/wup/Highlights/ WUP2014-Highlights.pdf

2 Bettencourt LMA, Lobo J, Helbing D, et al. Growth, innovation, scaling, and the pace of life in cities. Proc Natl Acad Sci U S A 2007:104:7301-6.

3 Khreis H, Warsow K, Verlinghieri E, et al. Urban transport and health: understanding real impacts, underlying driving forces and Co-Producing future directions. Journal of Transport \& Health 2016;3:S7-8.

4 Nieuwenhuijsen MJ. Urban and transport planning pathways to carbon neutral, liveable and healthy cities; a review of the current evidence. Environ Int 2020;140:105661.

5 Beelen R, Raaschou-Nielsen O, Stafoggia M, et al. Effects of longterm exposure to air pollution on natural-cause mortality: an analysis of 22 European cohorts within the multicentre escape project. Lancet 2014;383:785-95.

6 Héroux M-E, Anderson HR, Atkinson R, et al. Quantifying the health impacts of ambient air pollutants: recommendations of a $\mathrm{WHO} /$ Europe project. Int J Public Health 2015;60:619-27.

7 Basner M, Babisch W, Davis A, et al. Auditory and non-auditory effects of noise on health. Lancet 2014;383:1325-32.

8 Halonen JI, Hansell AL, Gulliver J, et al. Road traffic noise is associated with increased cardiovascular morbidity and mortality and all-cause mortality in London. Eur Heart J 2015;36:2653-61.

9 Gasparrini A, Guo Y, Hashizume M, et al. Mortality risk attributable to high and low ambient temperature: a multicountry observational study. Lancet 2015;386:369-75.
10 Nieuwenhuijsen MJ, Khreis H, Triguero-Mas M, et al. Fifty Shades of green: pathway to healthy urban living. Epidemiology 2017;28:63-71.

11 Gascon M, Triguero-Mas M, Martínez D. Green space and mortality: a systematic review and meta-analysis. Environ Int 2016;2:60-7.

12 Woodcock J, Franco OH, Orsini N, et al. Non-vigorous physical activity and all-cause mortality: systematic review and meta-analysis of cohort studies. Int J Epidemiol 2011;40:121-38.

13 , Forouzanfar MH, Alexander L, et al, GBD 2013 Risk Factors Collaborators. Global, regional, and national comparative risk assessment of 79 behavioural, environmental and occupational, and metabolic risks or clusters of risks in 188 countries, 1990-2013: a systematic analysis for the global burden of disease study 2013. Lancet 2015;386:2287-323.

14 Hänninen $\mathrm{O}$, Knol $\mathrm{AB}$, Jantunen $\mathrm{M}$, et al. Environmental burden of disease in Europe: assessing nine risk factors in six countries. Environ Health Perspect 2014;122:439-46.

15 Lelieveld J, Evans JS, Fnais M, et al. The contribution of outdoor air pollution sources to premature mortality on a global scale. Nature 2015;525:367-71.

16 Briggs D, Mason K, Borman B. Rapid assessment of environmental health impacts for policy support: the example of road transport in New Zealand. Int J Environ Res Public Health 2016;13:61.

17 Stassen KR, Collier P, Torfs R. Environmental burden of disease due to transportation noise in Flanders (Belgium). Transp Res $D$ Transp Environ 2008;13:355-8.

18 Mueller N, Rojas-Rueda D, Cole-Hunter T, et al. Health impact assessment of active transportation: a systematic review. Prev Med 2015;76:103-14.

19 Rojas-Rueda D, de Nazelle A, Tainio M, et al. The health risks and benefits of cycling in urban environments compared with CAR use: health impact assessment study. BMJ 2011;343:d4521.

20 Woodcock J, Tainio M, Cheshire J, et al. Health effects of the London bicycle sharing system: health impact modelling study. BMJ 2014;348:g425.

21 Tobías A, Recio A, Díaz J, et al. Health impact assessment of traffic noise in Madrid (Spain). Environ Res 2015;137:136-40.

22 Perez L, Trüeb S, Cowie H, et al. Transport-Related measures to mitigate climate change in Basel, Switzerland: a health-effectiveness comparison study. Environ Int 2015;85:111-9.

23 Malmqvist E, Lisberg Jensen E, Westerberg K, et al. Estimated health benefits of exhaust free transport in the city of Malmö, southern Sweden. Environ Int 2018;118:78-85.

24 Woodcock J, Edwards P, Tonne C, et al. Public health benefits of strategies to reduce greenhouse-gas emissions: urban land transport. Lancet 2009;374:1930-43.

25 Stevenson M, Thompson J, de Sá TH, et al. Land use, transport, and population health: estimating the health benefits of compact cities. Lancet 2016;388:2925-35.

26 Mueller N, Rojas-Rueda D, Basagaña X, et al. Urban and transport planning related exposures and mortality: a health impact assessment for cities. Environ Health Perspect 2017;125:89-96.

27 Mueller N, Rojas-Rueda D, Basagaña X, et al. Health impacts related to urban and transport planning: a burden of disease assessment. Environ Int 2017;107:243-57.

28 Thondoo M, Mueller N, Rojas-Rueda D, et al. Participatory quantitative health impact assessment of urban transport planning: a case study from eastern Africa. Environ Int 2020;144:106027.

29 Sohrabi S, Khreis H. Burden of disease from transportation noise and motor vehicle crashes: analysis of data from Houston, Texas. Environ Int 2020;136:105520.

30 Sohrabi S, Zietsman J, Khreis H. Burden of disease assessment of ambient air pollution and premature mortality in urban areas: the role of socioeconomic status and transportation. Int J Environ Res Public Health 2020;17:1166.

31 Mueller N, Rojas-Rueda D, Khreis H, et al. Changing the urban design of cities for health: the superblock model. Environ Int 2020;134:105132.

32 Mueller N, Rojas-Rueda D, Khreis H, et al. Socioeconomic inequalities in urban and transport planning related exposures and mortality: a health impact assessment study for Bradford, UK Environ Int 2018;121:931-41.

33 lungman T, Khomenko S, Nieuwenhuijsen M, et al. The impact of urban and transport planning on health: assessment of the attributable mortality burden in Madrid and Barcelona and its distribution by socioeconomic status. Environ Res 2021;196:110988

34 de Nazelle A, Nieuwenhuijsen MJ, Antó JM, et al. Improving health through policies that promote active travel: a review of evidence to support integrated health impact assessment. Environ Int 2011;37:766-77. 
35 Nieuwenhuijsen MJ. Urban and transport planning, environmental exposures and health-new concepts, methods and tools to improve health in cities. Environ Health 2016;15.

36 Glazener A, Sanchez K, Ramani T, et al. Fourteen pathways between urban transportation and health: a conceptual model and literature review. Journal of Transport \& Health 2021;21:101070.

37 Chen J, Hoek G. Long-Term exposure to PM and all-cause and cause-specific mortality: a systematic review and meta-analysis. Environ Int 2020;143:105974.

38 Huangfu P, Atkinson R. Long-term exposure to $\mathrm{NO}_{2}$ and $\mathrm{O}_{3}$ and all-cause and respiratory mortality: A systematic review and metaanalysis. Environ Int 2020;144:105998.

39 Kempen Evan, Casas M, Pershagen G, et al. Who environmental noise guidelines for the European region: a systematic review on environmental noise and cardiovascular and metabolic effects: a summary. Int J Environ Res Public Health 2018;15:379.

40 Eurostat. Urban audit, 2018. Available: https://ec.europa.eu/eurostat/ web/gisco/geodata/reference-data/administrative-units-statisticalunits/urban-audit

41 de Hoogh K, Chen J, Gulliver J, et al. Spatial PM2.5, NO2, O3 and bc models for Western Europe - evaluation of spatiotemporal stability. Environ Int 2018;120:81-92.

42 Thunis $\mathrm{P}$, Degraeuwe $\mathrm{B}$, Pisoni E, et al. PM2.5 source allocation in European cities: a SHERPA modelling study. Atmos Environ 2018;187:93-106.
43 Heydari S, Tainio M, Woodcock J, et al. Estimating traffic contribution to particulate matter concentration in urban areas using a multileve Bayesian meta-regression approach. Environ Int 2020;141:105800.

44 Eurostat. City statistics, 2019. Available: https://ec.europa.eu/ eurostat/web/cities/data/database

45 Eurostat. Eurostat database, 2019. Available: https://ec.europa.eu/ eurostat/data/databaseEurostat

46 Eurostat. Regional statistics by nuts classification, 2019. Available: https://ec.europa.eu/eurostat/web/regions/data/database

47 Khomenko S, Cirach M, Pereira-Barboza E, et al. Premature mortality due to air pollution in European cities: a health impact assessment. Lancet Planet Health 2021;5:e121-34.

48 Khreis H, de Hoogh K, Nieuwenhuijsen MJ. Full-chain health impact assessment of traffic-related air pollution and childhood asthma. Environ Int 2018;114:365-75.

49 Gulliver J, Morley D, Vienneau D, et al. Development of an open-source road traffic noise model for exposure assessment. Environmental Modelling \& Software 2015;74:183-93.

50 Mueller N, Daher C, Rojas-Rueda D, et al. Integrating health indicators into urban and transport planning: a narrative literature review and participatory process. Int J Hyg Environ Health $2021 ; 235: 113772$. 